Voix et Images

voixetimages

\title{
La quête d'identité : une aventure ambiguë
}

\section{Zila Bernd}

Volume 12, numéro 1 (34), automne 1986

Québec-Amérique latine

URI : https://id.erudit.org/iderudit/200603ar

DOI : https://doi.org/10.7202/200603ar

Aller au sommaire du numéro

Éditeur(s)

Université du Québec à Montréal

ISSN

0318-9201 (imprimé)

1705-933X (numérique)

Découvrir la revue

Citer cet article

Bernd, Z. (1986). La quête d'identité : une aventure ambiguë. Voix et Images, 12(1), 21-26. https://doi.org/10.7202/200603ar d'utilisation que vous pouvez consulter en ligne.

https://apropos.erudit.org/fr/usagers/politique-dutilisation/ 


\title{
La quête d'identité: une aventure ambiguë
}

\author{
par Zila Bernd, Université Fédérale du Rio Grande do Sul
}

$\boldsymbol{L}$ 'A venture ambiguë est le titre du roman de l'écrivain sénégalais, Cheikh Hamidou Kane, qui pourrait dire avec son président, Léopold Sédar Senghor: "Nous sommes des métis culturels. Si nous sentons en nègre, nous nous exprimons en français, parce que le français est une langue à vocation universelle". "Mais Cheikh $H$. Kane sait bien qu'il n'est pas toujours facile de choisir et que la 'tentation de l'Occident' ne se borne pas à la langue. $L$ 'A venture ambiguë est le récit d'un déchirement, de la crise de conscience qui accompagne, pour l'africain 'européanisé' sa propre prise de conscience ${ }^{1}$.

Le dénominateur commun des littératures des Amériques, y compris la québécoise, réside dans le fait que, dès leur naissance, elles se nourrissent de la sève, riche et sédimentée, de la culture européenne.

Mais aussi bien, ces littératures apparaissent stigmatisées par un rapport premier avec la culture des colonisateurs. C'est une relation où la réalité culturelle étrangère est perçue comme supérieure à la culture nationale ${ }^{2}$.

Il est vrai également que, depuis le début, les écrivains, consciemment ou non, ont pratiqué le marronnage culturel qui consiste à renverser les modèles importés et à incorporer au tissu littéraire l'apport des cultures autochtones. Dans ce sens, Eduardo Galeano ${ }^{3}$ associe la formation de la culture latinoaméricaine à un jeu de masques, métaphore de la tendance (manifeste dans la bourgeoisie et chez les élites) d'imiter les formules traditionnelles du Vieux Monde. Mais le livre de Galeano souligne aussi bien du côté des couches populaires la tendance à reproduire le vrai visage de l'Amérique par le sabotage de modèles altérés par des éléments culturels originels de l'Amérique.

Ce sera donc sous le signe de l'hybridisation que vont se construire les littératures des Amériques: d'une part, la présence de l'héritage européen et d'autre part, la désacralisation de cette culture et le dessein de recréer d'autres mythes - américains - sur lesquels pourra être fondée une littérature "authentique».

En effet, les chemins de cette (re)construction passent par la question de l'identité.

Darcy Ribeiro, dans une fable intitulée l'Utopie sauvage, pose les questions suivantes: Qui sommes-nous? Nous-mêmes? Eux? Personne ${ }^{44}$ Le fameux anthropologue brésilien souligne le fait que l'origine des maux des peuples latino-américains réside dans la perte de l'identité culturelle: $Q u i$ sommes-nous, si nous ne sommes plus européens, ni amérindiens, mais une espèce intermédiaire entre les autochtones et les européens? 
Ribeiro reprend les personnages de Shakespeare, Prospero et Caliban, dans une intertextualité d'ailleurs convoquée par Aimé Césaire dans Une tempête, et montre que la revanche de Caliban (le colonisé, l'esclave, l'exploité) consiste justement dans le processus d'affirmation de son identité. Dans l'Utopie sauvage, Caliban est un amérindien qui, par le rituel anthropophagique, mange les cendres de ses ancêtres pour trouver l'immortalité.

Notre objectif, ici, est d'aborder la question de l'identité comme trait commun entre les littératures des Amériques.

Effectivement, les problèmes concernant l'auto-définition de l'être deviennent la thématique obsessionnelle des principaux écrivains d'Amérique, y compris les Brésiliens et les Québécois. C'est dans cet esprit que nous tenterons de rapprocher l'œuvre de Jacques Godbout, les Têtes à Papineau ${ }^{5}$, de celle du Brésilien Moacyr Scliar, le Centaure dans le jardin'6, parues à une année d'intervalle (1981 et 1980).

\section{Le mythe du centaure}

Les deux auteurs jouent avec la métamorphose. Leurs personnages principaux sont des êtres anormaux, des créatures aberrantes. Dans le roman de Godbout, le personnage principal, Charles-François, a deux têtes et chez Scliar, il s'agit d'un centaure, appelé Guedali.

Les centaures sont des êtres monstrueux de la mythologie grecque, dont la tête, les bras et le buste sont d'un homme, le reste du corps et les jambes, d'un cheval. (...) Ils ont l'image frappante de la double nature de l'homme, l'une bestiale, l'autre divine?.

Dans la mythologie "gaucha" (du Rio Grande dol Sul, d'où Scliar est originaire), l'image du centaure est associée au gaucho qui, dans son habitat naturel, les champs où il s'adonne à l'élevage du bétail, dépend tellement du cheval qu'il finit par être évoqué, dans l'Histoire et la Littérature, comme le centaure de la pampa.

Le roman présénte un personnage, Guedali, issu d'une famille juive et né au sud du Brésil. Étonnement de la famille, lorsqu'elle s'aperçoit que le bébé est un centaure.

Évidemment, il s'agit d'un récit qui relève du fantastique, dont la trame tourne autour du désir de Guedali de redevenir «normal», homme parmi les hommes. Il se marie à une centauresse et tous deux découvrent l'existence d'un médecin qui les opérera pour les guérir de leur atroce condition. En effet, la chirurgie réussit et, chaussés de bottes, ils peuvent marcher - comme le reste des mortels - sur les deux pieds et non plus sur quatre pattes.

Après la métamorphose, ils réintègrent la vie normale: travail, famille, amis, vie sociale, etc. Pourtant, au niveau physique, subsistent des cicatrices, les sabots qui doivent être cachés par les bottes et, au niveau intellectuel, la nostalgie du temps où ils parcouraient librement les campagnes sur leurs propres pattes. 
Un jour, il décide de se faire réopérer pour redevenir centaure. Il part voir le médecin marocain qui a exécuté la première intervention, mais l'opération n'a pas lieu finalement. Il doit retourner au Brésil où angoissé, il s'interroge sur son existence: Est-ce que Guedali était un petit centaure heureux? Etait-il plus ou moins heureux que Guedali bipède? S'il était moins heureux pourquoi, donc, cette angoisse, ce besoin irrésistible de galoper, cette recherche incessante de quelque chose dont je ne connais pas très bien la nature? 8

L'introduction dans le récit de ces être légendaires - comme des sirènes, des sphinx qui apparaissent dans les autres romans de l'auteur - symbolisent l'ambivalence humaine en général et l'ambivalence des Juifs en particulier qui se sentent liés à deux cultures. Le titre même du roman est doublement ambigu: d'un côté la double nature du centaure et, de l'autre, ce monstre (= sauvagerie) emprisonné dans un jardin (= espace civilisé).

Chez Scliar, la dichotomie entre l'appartenance à l'Amérique et les origines juives va engendrer le motif du centaure, en permanente quête d'identité.

À l'origine, le mythe naît dans les moments de crise comme tentative de remplir l'espace crée par la rupture entre l'homme et la nature. Le mythe est la tentative de dépasser la perte d'unité originelle avec la nature ou représente la forme d'interpréter l'union avec la totalité 9 . Si le discours mythique, dans les sociétés primitives et archaïques, se construit pour dépasser une rupture et participe à la réorganisation du cosmos, à l'ordonnancement de l'univers, les cuvres actuelles élaborent à leur tour leur propre mythologie pour tenter de résoudre une crise d'identité.

Le centaure est donc le symbole, à la fois de la bipartition de l'être entre l'animique et le matériel, mais aussi de la bipartition culturelle entre l'héritage culturel du Vieux Monde et l'émergence d'une culture propre à l'Amérique.

\section{Le bicéphalisme}

En ce qui concerne l'auteur québécois, le ton général de l'œuvre est plus ironique et même plus humoristique que chez l'écrivain brésilien. Pourtant les deux récits plongent le lecteur dans une atmosphère fantastique relevant d'une genèse commune: la prise de conscience de la nature hétérogène de la formation ethnique et culturelle des Amériques.

Chez Godbout, les deux têtes autonomes prennent racine à la hauteur de la trachée-artère comme les deux branches d'un V victorieux ${ }^{10}$. CharlesFrançois, dit "Les Têtes», veulent redevenir "normaux», mais leur principale préoccupation consiste à savoir s'ils vont garder la mémoire après l'opération. Le docteur les rassure: il n'est pas là pour les anéantir, il est tout à fait conscient de la réalité, Charles et François ont chacun leur vision du monde.

Comme le note Eunice Galery, les noms des têtes constituent déjà une piste: Charles signifie "homme» et François, "français»" Outre la référence historique, Papineau, diminutif de Papin, signifie bouillie ou mangeur de 
bouillie, et encore écraser, éventrer, démolir. Les personnages charrient donc, dans leur nom, leur destinée: homme français écrasé, éventré, démoli.

Par sa forme sphérique, la tête représente l'univers. C'est un microcosme. Le bicéphalisme peut alors être vu comme symbole de deux microcosmes, deux visions du monde, mais inséparables puisqu'appartenant à un même corps. Dans cette mesure, ce n'est pas par hasard que l'auteur crée l'image du siamois (qui, ironiquement, rime avec québécois...):

Les mythologies font toutes allusions à des êtres polycéphales: animaux, hommes, génies, dieux, déesses. Chacune de ces têtes est une des manifestations particulières de l'UN; par exemple, un dieu tricéphale exprime trois aspects de la puissance ${ }^{12}$.

Mais le phénomène du bicéphalisme doit également être interprété en rapport avec le bilinguisme des Canadiens français. Les deux têtes expriment aussi, de façon symbolique, la question de l'auto-référentialité qui se posait aux Québécois. Le problème de l'identité apparaît déjà dans l'acte de se nommer. Deux mots sont nécessaires: «canadien» et "français» (du moins avant l'adoption du vocable "québécois"). L'hybridisme est à l'origine même de leur double nom: le premier les rattache à l'Amérique, le second aux ancêtres gaulois.

Alain Piette a déjà signalé la pluralité d'interprétation que permet ce roman caractérisé par l'entrecroisement des discours. Selon son analyse, l'hésitation des Québécois entre le OUI et le NON, au moment du référendum de 1980 , sous-tend le texte qui peut donc être lu comme la réécriture du texte national ${ }^{13}$.

En 1968, Pierre Vallières créait l'expression «nègres blancs d'Amérique" pour désigner le Québécois qui, par sa condition historique, fait quotidiennement l'expérience de sa propre précarité. Il sait - comme le JUIF, le Tzigane et le Palestinien - qu'il possède ni patrie ni destin ni survie assurés. Son histoire n'est pas derrière lui, mais devant. Il se doit de l'inventer, sous peine de disparaitre au prochain grand tournant ${ }^{14}$.

C'est cette survie menacée, ce futur incertain des Québécois qui inspire dans les Têtes la thématique de la métamorphose. Comme le centaure, les têtes sont obsédées par l'idée de se transformer.

Selon la mythologie, ces changements n'affectent pas, toutefois, les personnalités profondes qui gardent, en général, leur nom et leur psychisme. D'un point de vue analytique, les métamorphoses sont des expressions du désir, de la censure, de l'idéal, de la sanction, issus des profondeurs de l'inconscient et prenant forme dans l'imagination créatrice ${ }^{15}$. En tant qu'expression du désir et de l'idéal, la métamorphose devient, en effet, caractéristique d'une phase où l'individu n'a pas encore actualisé la totalité de ses capacités potentielles.

Il paraît évident chez nos deux auteurs que le désir de leurs personnages, relativement à la métamorphose, symbolise la conscience de l'homme améri- 
cain dont l'identité reste encore mal définie, en procès de constitution. À la limite, le déchirement des personnages qui veulent devenir autres, représente le processus de transculturation, qui est le propre du continent américain, toujours en quête de sa propre définition.

Si la métamorphose est dynamique, l'amalgame (mélange d'éléments divers qui vont constituer une totalité nouvelle) est définitif et irréversible. Désireuses de changer, de devenir unicéphales, les têtes sont soumises à une intervention chirurgicale qui réalisera un amalgame: la combinaison par le Dr. Northridge de la moitié gauche du cerveau d'une des têtes et de la moitié droite de l'autre. Le résultat est l'Amalgamé, être unicéphale, qui devient aussi unilingue. Il perd sa capacité de s'exprimer en langue française: on assiste à la naissance d'un autre monstre.

Scliar se met sous le signe dynamique de la métamorphose, tandis que Godbout met en garde contre l'amalgame qui détermine nécessairement la perte ou l'annulation d'un des éléments constitutifs.

\section{Les signes en rotation}

Par rapport aux littératures américaines, nous ne pouvons plus - depuis longtemps déjà - parler de dépendance culturelle. Nous vivons sous le signe de la métamorphose, et les monstres que nous rencontrons ne constituent peut-être pas des cas tératologiques: il faut que la diversité, la pluralité des formes soient envisagées comme des richesses plutôt que comme des aberrations.

A une conception monolithique de l'identité doit répondre une conception pluri-dimensionnelle du sujet. L'image d'une mosaïque, formée de fragments d'origines diverses qui ne perdent nullement leurs particularités en formant une nouvelle totalité, sera peut-être la plus convenable pour rendre compte de cette question si complexe.

En effet, les diverses dimensions de l'être américain doivent être prises en compte, sans exclusive. C'est ainsi que chez Scliar le problème ne se réduit pas à la recherche de sa condition juive: il veut aussi bien assumer sa gauchité ${ }^{16}$, sa brésilianité, son américanité.

La question de l'identité fait corps avec celle de l'altérité, le sentiment de l'autre, idée selon laquelle l'être est impossible hors des relations qui le tient à l'autre.

Ni MANIE, ni PHOBIE mais PHILIE qui autorise les échanges bilatéraux et l'admiration mutuelle. La réalité culturelle étrangère est positive, mais la culture nationale l'est également, offrant les conditions idéales d'un vrai dialogue interculturel.

Les êtres monstrueux qui habitent l'imaginaire de ces deux grands auteurs des Amériques ne doivent pas être considérés comme anormaux. Cette hybridité devient l'image d'un exil du personnage: il vit l'expérience de 
l'exil à l'intérieur de soi-même et de son propre pays, à cause d'une nature qui le met à l'écart des autres.

Il faut pourtant admettre que cette double nature recèle aussi un pouvoir: Janus, roi légendaire du Latium, a deux têtes, ou plutôt deux visages, qui lui octroient le pouvoir de voir en avant et en arrière, le passé et l'avenir. Le centaure a des attributs (comme la force physique) que l'homme n'a pas. Dans cette mesure, nous pouvons conclure, en nous inspirant des réflexions d'Octavio $\mathrm{Paz}^{17}$, que les signes sont dans un perpétuel mouvement de rotation; les signes qui nous exilent sont les mêmes que ceux qui nous constituent dans notre humaine condition, ce qui est à la fois notre fortune et notre damnation.

1. V. Monteil, préface de l'Aventure ambiguë de C. H. Kane, Paris. Union Générale d'Éditions, 1961.

2. Cf. la notion de Manie, selon la terminologie de D. H. Pageaux \& A. M. Machado in Literatura portuguesa, literatura comparada e teoria da literatura, Lisboa, Ediçoes 70,1981 , p. 50.

3. E. Galeano, As caras e as mascaras, Sao Paulo, Nova Fronteira, 1985.

4. D. Ribeiro, Utopia Selvagem, Rio de Janeiro, Nova Fronteira, 1982, p. 32.

5. J. Godbout, les Têtes à Papineau, Paris, Seuil, 1981.

6. M. Scliar, le Centaure dans le jardin. Paris, Presses de la Renaissance, 1985 (la première édition brésilienne date de 1980).

7. J. Chevalier et A. Gheerbrant, Dictionnaire des symboles, Paris, Seghers, 1974, 4v., p. 299 (A-C).

8. M. Scliar, op. cit., p. 232.

9. S. Y. Machado, O labirinto em Moacyr Scliar, Porto Alegre, UFRGS, 1983 (Thèse de Mestrado, mimeo), Introduction, p. IV.

10. J. Godbout, op. cit., p. 15.

11. E. Galery, "Comment peut-on être québécois?", Études littéraires, Québec, PUL, 16 (2), 1983, p. 225.

12. J. Chevalier et A. Gheerbrant, op. cit., p. 288 (P-Z).

13. A. Piette, "Les langues à Papineau: comment le texte national se fait littérature». Voix et images, Montréal, IX (3), printemps 1984, p. 114-127.

14. P. Vallières, Nègres blancs d'Amérique, Québec, Parti pris, 1968, p. 22.

15. J. Chevalier et A. Gheerbrant, op. cit., p. 212 (H-P).

16. "Gaucho": Brésilien du sud du pays qui revendique son identité avec fierté sans aucun sentiment de rejet par rapport à l'identité brésilienne.

17. O. Paz, O arco e a lira (El arco y la lira). Rio de Janeiro, Nova Fronteira, 1982, p. $309-350$.

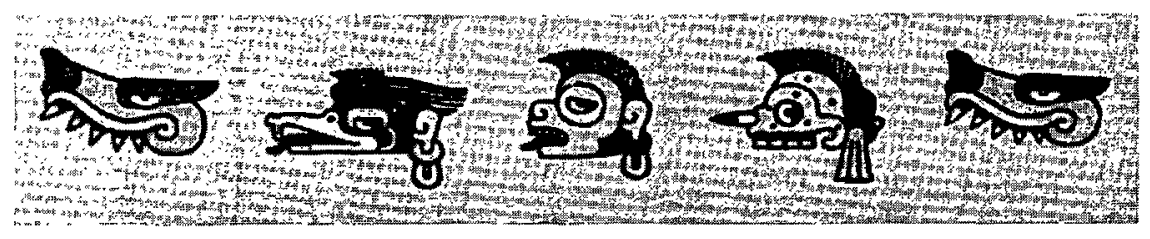

